\title{
EFICIÊNCIA ENERGÉTICA EM SISTEMAS DE ELEVAÇÃO ARTIFICIAL - BOMBEIO MECÂNICO
}

\author{
I. C. BASTOS \\ Universidade Federal de Alfenas, Instituto de Ciência e Tecnologia \\ Izabella.carneiro@unifal-mg.edu.br*
}

Submetido 10/10/2016 - Aceito 17/02/2017

DOI: $10.15628 /$ holos.2017.5194

\section{RESUMO}

Quando a própria pressão do poço não é suficiente para elevar seus fluidos de forma economicamente viável se faz necessário à utilização de algum método de elevação artificial. O bombeio mecânico com hastes é o método de elevação mais utilizado no mundo, por isso conhecer seu funcionamento e atribuir técnicas para aumentar sua eficiência são de extrema importância. Estima-se que $90 \%$ da elevação artificial no mundo utilize o sistema de bombeio mecânico.

Embora este sistema seja considerado o mais antigo e o mais popular método de elevação artificial, ainda existem estudos sobre a quantidade de energia consumida e quantidade de óleo/gás produzido. Pesquisas têm mostrado, por exemplo, que as unidades de hastes de bombeio apresentam uma eficiência energética de apenas $50 \%$.

Uma vez que a maioria das instalações é acionada por um motor elétrico e ainda que o custo de energia elétrica esteja aumentado gradativamente, a eficiência energética tornou-se o elemento mais decisivo para as despesas operacionais em campos bombeados por este tipo de sistema.

Este trabalho apresenta técnicas de otimização através da redução das perdas de energia no bombeio mecânico. Uma visão geral relativa à eficiência do sistema, a redução de custo de operação e as melhores praticas para maximizar a produção e consequentemente a eficiência serão apresentados.

PALAVRAS-CHAVE: Sistema de Bombeio Mecânico, Eficiência Energética, Monitoramento.

\section{ENERGY EFFICIENCY IN ARTIFICIAL LIFT SYSTEMS - SUCKER ROD PUMPING SYSTEM}

\begin{abstract}
When the inherent pressure is not enough to raise the fluids economically viable it is necessary to use some method of artificial lift. The mechanical pumping with rods is the most common lifting method in the world, knowledge of operation and techniques to increase efficiency is essential. It is estimated that $90 \%$ of artificial lift in the world are using the mechanical pumping system. Research has shown, for example, that the sucker rods drives usually have an efficiency of only $50 \%$. Since most of the facilities are driven by an electric
\end{abstract}

motor and the costs of electricity is continuously increasing, energy efficiency has become the most decisive element for operating expenses for this type of system.

This paper presents optimization techniques by reducing energy losses in the mechanical pumping system. An overview of the system efficiency, a possible reduction of operational costs, best practices to maximize production, and new technologies for their effectiveness are presented.

KEYWORDS: Sucker Rod Pumping System, Energy Efficiency, Monitoring, 


\section{INTRODUÇÃO}

Poços de petróleo que no início de sua vida produtiva possuem energia suficiente para que os fluidos sejam produzidos naturalmente são chamados poços surgentes. Isto ocorre devido à pressão no fundo do poço ser superior a todas as perdas de pressão que o fluido sofre ao longo da elevação. Quando esta pressão começa a diminuir, a diferença entre a pressão no fundo e todas as perdas de pressão começa a cair, causando uma diminuição do fluxo que chega à superfície. Para que estes poços voltem a produzir ou até mesmo para aumentar a produção de poços surgentes, métodos de elevação artificial são utilizados. Esses métodos atuam fornecendo energia adicional ao poço necessária para elevar os fluidos. Um dos métodos de elevação artificiais mais utilizados mundialmente é o Bombeio Mecânico (BM). Este sistema é a forma mais comum de elevação artificial e será considerando neste trabalho. Estima-se que $90 \%$ utilize o sistema de bombeio mecânico para aumentar a produção de poços [1-4].

O grande sucesso do sistema de bombeio com hastes está relacionada ao investimento de baixo custo, manutenção e flexibilidade para operar com fluidos de várias composições e com um grande intervalo de temperatura. Contudo, o uso ineficiente da energia é um dos problemas operacionais mais comuns experimentados a partir deste sistema de elevação artificial. Devido às ineficiências dos vários componentes, perdas de energia ocorrem durante a elevação do fluido para a superfície. Um dos exemplos esta relacionado ao preenchimento incompleto da bomba de fundo sendo a razão mais comum para a baixa eficiência de produção e normalmente a maior fonte de desperdício de energia.

Devido à tendência mundial de menor consumo de energia e, portanto, redução de perdas de energia, a eficiência energética tornou-se o elemento mais decisivo para as despesas operacionais em campos que utilizam este tipo de sistema. Operações mais eficientes e com menor consumo de energia resultará consequentemente na otimização do sistema. Portanto, este trabalho irá definir exatamente as áreas problemáticas e sugestões para otimizar o sistema do ponto de vista da eficiência energética. Para isso, uma análise completa do sistema será realizada considerando pontos que podem influenciar significativamente em perdas de energia e consequentemente em seu desempenho. Além disso, a medida que a unidade de bombeio já está instalada no campo, a otimização obtida minimizará todos os custos de operação envolvidos.

\section{Sistemas de Bombeio Mecânico}

O bombeio mecânico é a forma mais comum de elevação artificial e é amplamente utilizada em aplicações onshore, seja devido ao baixo custo de investimento ou sua capacidade de produção ao longo da vida produtiva do poço. Existe uma divisão básica de componentes no BM: Os componentes de superfície e os componentes de fundo [1]. Os componentes de superfície são: o motor (prime mover), a caixa de redução (gear box), a unidade de bombeio (pumping unit) e a haste polida (polished rod). Já os componentes de fundo são: a coluna de hastes (rod string) e a 
bomba de fundo (downhole pump). A Figura 1 exibe as principais partes de um sistema de bombeio mecânico de superfície.

Esta bomba convencional tem um design mecânico muito complexo cujo princípio de funcionamento é baseado no movimento rotativo de um motor elétrico o qual é transformado em movimento alternativo pela unidade de bombeio. Uma coluna de hastes transfere o movimento para a parte inferior que aciona uma bomba capaz de elevar os fluidos do reservatório até à superfície. A bomba em si é constituída por um cilindro, também chamado de barril, com válvulas que serão acionadas para deslocar o fluido. As válvulas consistem em um anel de vedação e uma esfera que é movida pelo movimento da bomba em combinação com a pressão do fluido. A extremidade superior da coluna de hastes está ligada a uma haste polida que está em movimento alternativo acionado pela unidade de bombeamento. Para entender todo o conceito é útil comparar o sistema de hastes de bombeio com uma linha de transmissão. $\mathrm{Na}$ extremidade superior há o receptor e na extremidade inferior o transmissor. Informações sobre o fundo de poço e a condição bomba é transmitida ao longo das hastes de bombeio e monitorada na superfície por um dinamômetro [5].

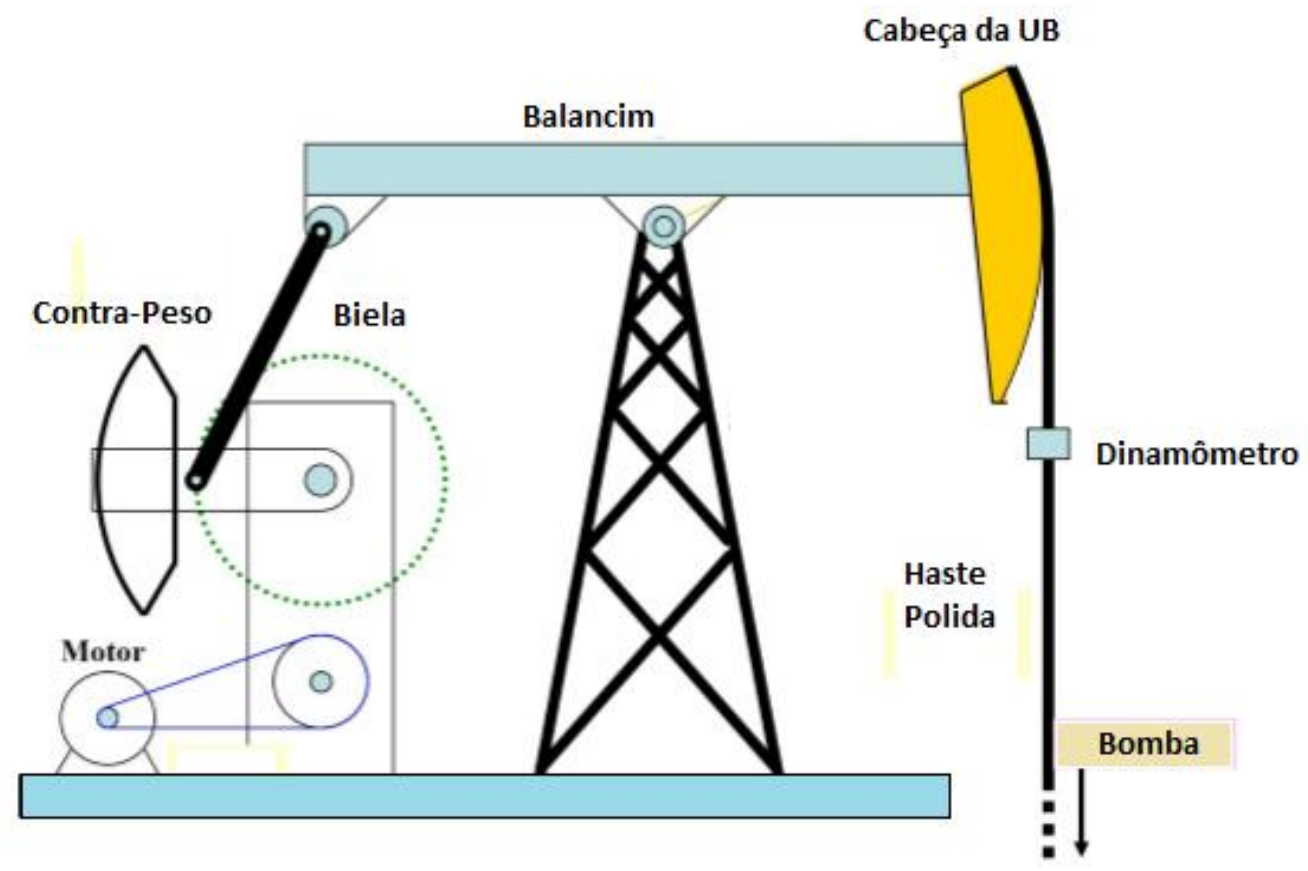

Figura 1: Principais partes do sistema de bombeio de superfície.

O dinamômetro corresponde a maioria das técnicas de diagnóstico atuais para bombeamento mecânico e é baseado nas medições de carga impostas por todo o sistema. Aplicado diretamente sobre a haste polida sua função é medir simultaneamente a carga e o movimento (posição) da haste durante a operação do sistema (carta dinamométrica de superfície) [5]. Alguns softwares já preveem o comportamento do sistema baseado em suas configurações mecânicas, seja no tamanho da própria unidade de bombeio mecânico de superfície ou até mesmo no tipo de material da coluna de hastes e assim por diante. 
Portanto, a fim de aumentar o uso eficiente de energia no sistema, uma das técnicas utilizadas corresponde ao monitoramento ou até mesmo, uma pré - análise matemática para compreender o comportamento do tipo de bomba e, mais tarde, utilizar este conhecimento no campo. Esta bomba é um sistema mecânico muito complexo que integra as condições de fundo de poço e de superfície, como por exemplo, a energia necessária para levantar o óleo até a superfície, em outras palavras, o requisito de energia e os custos de energia para tornar a elevação possível.

\section{Eficiência Energética Sistemas de Bombeamento Mecânico}

A utilização eficiente da energia é hoje em dia uma tarefa muito importante. Em tempos em que o aquecimento global e as mudanças climáticas são motivo de preocupação no mundo, a melhoria da eficiência energética é a solução mais econômica, eficaz e rápida para minimizar impactos ambientais acarretados pela utilização da energia e reduzir emissões de dióxido de carbono $\left(\mathrm{CO}_{2}\right)$. O objetivo principal do uso racional de energia ou eficiência energética é reduzir a quantidade de energia necessária para o menor nível possível alcançando o mesmo resultado, seja aplicado em produtos, processos ou serviços. Melhorar a eficiência energética está muitas vezes relacionada com a utilização de novas ou tecnologias mais eficientes.

A indústria de Petróleo usa uma grande quantidade de energia para alimentar uma variada gama de processos de fabricação e de extração de recursos. Muitos dos processos industriais exigem grandes quantidades de calor e energia mecânica. Considerando a redução drástica do preço do petróleo nos últimos tempos e o contínuo uso deste produto, medidas devem ser tomadas para que a produção continue atendendo as expectativas do mercado. Neste sentido, a eficiência energética aplicada aos métodos de produção podem se tornar uma chave para redução de custos, aumento da competitividade e consequentemente melhoras no desempenho econômico das empresas petrolíferas. Como os processos industriais são diversos, é difícil descrever a multiplicidade de possíveis oportunidades de eficiência energética neste tipo de indústria. Neste caso será selecionado o método de elevação artificial mais utilizado em termos de número de instalações em todo mundo. Estima-se que $90 \%$ utilize o sistema de bombeio mecânico para aumentar a produção de poços. No Brasil, $64 \%$ de todos os poços produtores utilizam este sistema. Nos EUA, $85 \%$ dos poços artificialmente levantados são também produzidos por bombas de haste. $\mathrm{O}$ baixo custo, a simplicidade mecânica e a facilidade com que a operação seja executada, fazem com este tipo de bomba continue sendo utilizada indicando o seu domínio em operações onshore.

Embora as unidades de hastes de bombeio, em termos de produção, são considerados os meios mais eficientes dos métodos de elevação artificial, eles ainda são altamente ineficientes em termos dos valores reais da energia consumida. Estudos têm demonstrado que hastes de bombeio têm uma eficiência energética de cerca de apenas $50 \%$. Nas situações em que seu funcionamento é baseado em um motor elétrico, apenas $50 \%$ da energia elétrica consumida é convertida em potência hidráulica, com o restante sendo perdido em atrito ou outras ineficiências. Para obter o desempenho do sistema, uma análise integrada envolvendo o motor, equipamentos de superfície e equipamentos do fundo do poço (coluna de produção e bomba de fundo) serão realizados. A 
Figura 2 demonstra o fluxo de energia no sistema de bombeamento mecânico [2-5]. Observa-se pela própria figura que a eficiência energética dos componentes do sistema de bombeamento pode ser caracterizado pela quantidade relativa de perdas de energia. Portanto, o sistema pode ser divido em três partes sendo definidas como perdas de energia do fundo do poço, perdas da bomba de superfície e perdas relacionadas ao motor elétrico [1-5].

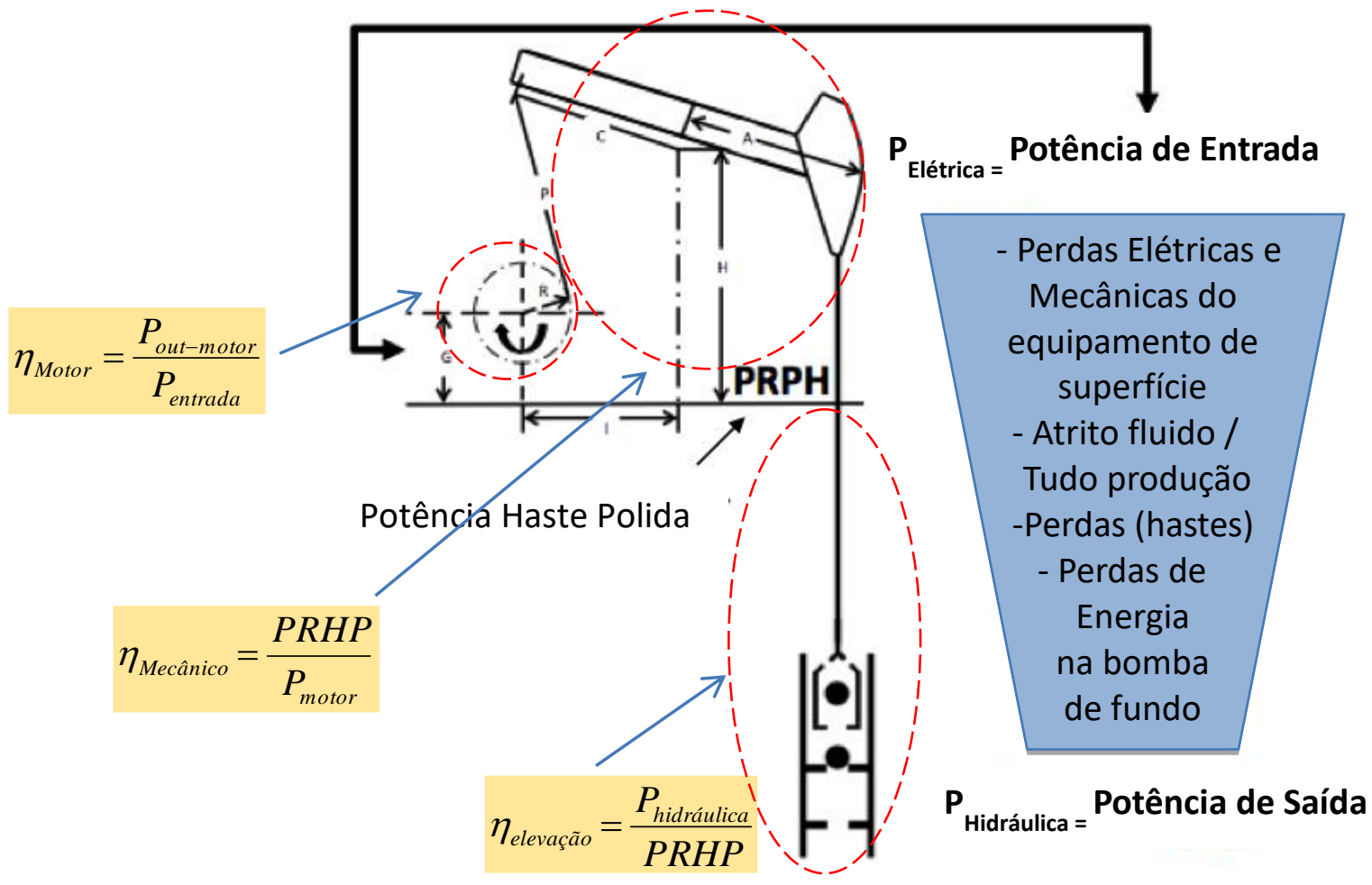

Figura 2: Perda de Energia no sistema[3].

Como a maioria das aplicações onshore exigem motores elétricos e o custo da energia elétrica tem aumentado ao longo dos anos, as perdas de energia devem ser minimizadas. Como demonstrado na Figura 2, o motor primário é responsável pela entrada de potência para guiar o sistema. Uma coluna de hastes transfere o movimento para parte inferior do sistema, que aciona uma bomba capaz de elevar os fluidos do reservatório produzidos até à superfície. Esta potência de entrada cobre toda a potência necessária do sistema para elevar o líquido e superar as perdas de energia decorrente do processo. A potência de saída, potência hidráulica, é caracterizada pelo trabalho realizado pela bomba para a elevação de uma dada quantidade de líquido para a superfície. Assumindo que o sistema não possua nenhum bloqueio ou fuga de gás, um cálculo teórico pode ser apresentado. Partindo disto é possível calcular a eficiência total do sistema ( sistema) sendo [2-6]:

$$
\eta_{\text {sistema }}=\frac{P_{\text {hidráulica }}}{P_{\text {entrada }}}
$$


Como os componentes do sistema de bombeamento mecânico estão conectados em série e as perdas de energia decorrem de diferentes naturezas, o cálculo da eficiência pode ser mais bem representado separando os componentes e calculando as eficiências individualmente. Sabese que a unidade de bombeio é um sistema complexo e que algumas perdas de energia não podem ser calculadas. Portanto, uma aproximação da eficiência do sistema pode ser demonstrada através do cálculo da eficiência de elevação, eficiência mecânica e a eficiência do motor elétrico.

A eficiência de elevação pode ser calculada considerando a quantidade de perda de energia do fundo do poço até a haste polida e pode ser representada como:

$$
\eta_{\text {elevação }}=\frac{P_{\text {hidráulica }}}{P_{\text {PRHP }}}
$$

Onde PRHP [W] corresponde à potência da haste polida e é representada pela quantidade de energia necessária para sobrepor a perda de energia decorrente do fundo do poço seja devido às perdas por atrito entre a coluna de fluido e as hastes de bombeio, perdas hidráulicas da própria bomba de fundo e etc. A potência PRHP pode ser calculada pela área da carta dinamométrica a ser medida. Isto é possível, pois o produto da força e a distância (trabalho mecânico) é representado pela haste polida durante o ciclo de bombeio. A eficiência mecânica pode ser determinada relacionando a potência da haste polida e a potência do motor fornecida ao sistema e esta relacionada a bomba de superfície completa, sendo representada por [2-6]:

$$
\eta_{\text {mecânico }}=\frac{P_{\text {PRHP }}}{P_{\text {motor }}}
$$

Observa-se que o rendimento mecânico da superfície corresponde apenas à entrada da caixa de engrenagem iniciando sua conexão com unidade de bombeio de superfície. Isto está relacionado com a potência necessária no eixo do motor e a potência dada até a haste polida (PRHP). As informações relativas à potência necessária no eixo do mecanismo principal não está disponível, no entanto, mais detalhes podem obtidos através da curva de eficiência a ser fornecida pelo próprio fabricante do motor. A eficiência do motor é o parâmetro mais importante que pode ser calculado. A eficiência energética do sistema está diretamente relacionada com a escolha e o funcionamento do motor [2]. Os custos de energia são uma parte significativa dos custos de operação do sistema e sua eficiência pode ser descrita pela potência de entrada necessária para suprir todo o sistema e a potência dada no eixo do motor. Sendo assim, a eficiência do motor pode ser calculada como [2-6]: 


$$
\eta_{\text {motor }}=\frac{P_{\text {motor }}}{P_{\text {entrada }}}
$$

No motor elétrico, as perdas de energia podem ser separadas em perdas mecânicas, atritos e perdas elétricas que são consideradas como a soma das perdas no núcleo, perdas no cobre ou enrolamentos e outras perdas adicionais. Em campo, o motor elétrico utilizado é do tipo NEMA D devido ao alto torque oferecido e alto valor de escorregamento.

\section{Técnicas para Otimização do Sistema}

Considerando os cálculos das principais eficiências, é possível encontrar a eficiência ótima do sistema de bombeamento mecânico podendo ser definida por [2-6]::

$$
\eta_{\text {sistema }}=\eta_{\text {elevação }} \eta_{\text {mecânico }} \eta_{\text {motor }}
$$

Com esta descrição, a melhoria da eficiência é realizada alterando algumas partes da equação. $\mathrm{O}$ único termo, o que permite maior número de modificações, é a eficiência de elevação $\left(\eta_{\text {elevação }}\right)$. Neste caso, a velocidade da unidade de bombeamento, o tamanho do êmbolo e o comprimento do curso podem ser alterados obtendo-se assim maior produtividade. Outro ponto que pode interferir na eficiência do sistema é a operação do motor elétrico e a caixa de engrenagens. Neste caso, se os componentes funcionam perto da sua capacidade nominal, a eficiência mecânica pode ser melhorada. No entanto, uma das maiores perdas de energia está relacionada ao sobre dimensionamento do motor elétrico em bombas já instaladas.

A meta ideal, utilizando o sistema de bombeamento, é sempre maximizar a produção e ao mesmo tempo obter os mais baixos custos de produção possíveis. A fim de atingir a melhor eficiência da unidade de bombeamento, algumas técnicas devem ser seguidas como:

- Atender a capacidade da unidade de bombeio com o desempenho do poço. O tamanho do pistão, velocidade de bombeio, comprimento do stroke, etc;

- Durante a produção, manter uma eficiência volumétrica elevada;

- Eliminar interferências de gás;

- Obter sempre a unidade de bombeamento balanceada (mecânica e eletricamente)

- Dimensionar corretamente o motor do sistema de bombeio de superfície: a maioria das unidades de bombeamento têm motores sobredimensionados.

- Não sobrecarregar a caixa de engrenagens e a própria unidade de bombeio de superficie. 0 uso correto do modelo da unidade de bombeio e hastes irá resultar em uma instalação mais eficiente e menos reparos durante o tempo de produção. 
- Aumentar a velocidade de bombeamento e utilizar hastes leves (FG) podem maximizar a produção e melhorar também a eficiência.

A operação de uma unidade de bombeamento é em grande parte dependente da qualidade do sistema de balanceamento. Para isso, é indispensável compreender os componentes necessários para equilibrar o sistema. Considerando-se as condições ideais de contrapeso, alguns benefícios podem ser alcançados, tais como: redução do tamanho da caixa de velocidades em relação a uma condição desequilibrada; redução do tamanho do motor elétrico; baixo custo de manutenção e aumento do tempo de vida útil do equipamento [7-10]. Uma maneira fácil de verificar se a unidade esta equilibrada consiste em medir a corrente do motor por ciclo. Para isto, a corrente versus posição da haste polida por ser obtida. Se o valor de pico no curso ascendente é o mesmo que o valor de pico no curso descendente, a unidade esta equilibrada. Esta visão geral é importante para entender como o contrapeso é indispensável para o funcionamento do sistema.

Outros pontos a serem melhorados são mais específicos e estão relacionados com as condições do fundo de poço e devem ser analisados caso a caso. Na tabela 1 tem-se um exemplo de técnicas que podem ser utilizadas para a melhoria da eficiência do sistema durante sua operação.

\begin{tabular}{|c|c|c|c|}
\hline $\begin{array}{c}\text { Situação do } \\
\text { Poço }\end{array}$ & $\begin{array}{c}\text { Baixa Produção ou Nível } \\
\text { Baixo da de Fluido }\end{array}$ & $\begin{array}{c}\text { Baixa Produção ou Nível } \\
\text { Baixo do Fluido }\end{array}$ & $\begin{array}{c}\text { Alta Produção ou Nível Alto } \\
\text { do Fluido }\end{array}$ \\
\hline $\begin{array}{c}\text { Leitura via cartas } \\
\text { dinamométricas }\end{array}$ & Bomba completa & $\begin{array}{c}\text { Baixo preenchimento da } \\
\text { bomba }\end{array}$ & $\begin{array}{c}\text { Baixo preenchimento da } \\
\text { bomba }\end{array}$ \\
\hline Trabalho do Engenheiro & $\begin{array}{c}\text { Baixa eficiência > Estudo da } \\
\text { Eficiência da superfície } \\
\text { Vazamento no tubo? }\end{array}$ & $\begin{array}{c}\text { Melhorar / adicionar controle } \\
\text { do poço durante a operação }\end{array}$ & $\begin{array}{c}\text { Alta prioridade - Estudo da } \\
\text { interferência de gás }\end{array}$ \\
\hline
\end{tabular}

Tabela 1: Exemplo de técnicas para melhoria do sistema $[8,9]$.

\section{Conclusão}

O sistema de elevação artificial mais popular é tradicionalmente o sistema de bombeio mecânico e cerca de $90 \%$ de todos os poços de elevação artificial estão equipados por esta bomba. Este trabalho descreve os primeiros passos para melhorar o desempenho do sistema em relação a sua eficiência energética. O sistema de bombeamento é complexo e para obter o melhor projeto para uma determinada aplicação, cada variável deve ser avaliada em termos das necessidades específicas do sistema a ser utilizado. 


\section{Referências}

Takács, G. Sucker-Rod Pumping Manual. Penn Well Corporation, 2003.

Economides, M. J., Hill, A. D. and Economides, C.E. Petroleum Production Systems. Prentice Hall PTR, 1993.

Takács, G. Ways to Obtain Optimum Power Efficiency of Artificial Lift Installations. SPE 126544, Society of Petroleum Engineers, January 2010.

Shedid, A. Technical and Economical Optimization of the Performance of sucker Rod Pumping System, Petroleum Technology Development Journal, Vol.2, July 2012.

Nascimento, J. M. A., Simulador Computacional para Poços de Petróleo com Método de Elevação Artificial por Bombeio Mecânico. (Tese de M. Sc. PPGEEC/UFRN), Natal, RN, Brasil, 2005.

Rischmüller, H., Meier, H. Oil Production with Subsurface Sucker Rod Pumps. Schoeller Bleckmann GmbH, January, 1989.

Skinner, D.R., Efficient Use of Electric Power in Production Operations, Journal of Petroleum Technology, Society of Petroleum Engineers of AIME, 1984.

Takacs, G., Power Efficiency of Sucker Rod Pumping, Petroleum Engineering Department, University off Miskolc.

Takacs, G., Ways to Decrease Production Costs for Sucker Rod Pumping, Petroleum Engineering Department, University off Miskolc.

[10] Takacs, G., Ways to Obtain Optimum Power Efficiency of Artificial Lift Installations, SPE 126544, January 2010. 